\title{
Seismicity Characterization and Monitoring at WESTCARB's Proposed Montezuma Hills Geologic Sequestration Site
}

\author{
T.M. Daley ${ }^{1}$, R. Haught, J.E. Peterson ${ }^{1}$, K. Boyle ${ }^{1,}$ J.H. Beyer ${ }^{1}$ and L.R. Hutchings ${ }^{1}$ \\ ${ }^{1}$ Lawrence Berkeley National Laboratory
}

\section{Introduction}

The West Coast Regional Carbon Sequestration Partnership (WESTCARB), in collaboration with Shell Oil Co. performed site characterization for a potential small-scale pilot test of geologic sequestration of carbon dioxide (CO2). The site area, know as Montezuma Hills, is near the town of Rio Vista in northern California. During the process of injection at a $\mathrm{CO}_{2}$ storage site, there is a potential for seismic events due to slippage upon pre-existing discontinuities or due to creation of new fractures. Observations from many injection projects have shown that the energy from these events can be used for monitoring of processes in the reservoir. Typically, the events are of relatively high frequency and very low amplitude. However, there are also well documented (non-CO2-related) cases in which subsurface injection operations have resulted in ground motion felt by near-by communities. Because of the active tectonics in California (in particular the San Andreas Fault system), and the potential for public concern, WESTCARB developed and followed an induced seismicity protocol (Myer and Daley, 2010). This protocol called for assessing the natural seismicity in the area and deploying a monitoring array if necessary. In this report, we present the results of the natural seismicity assessment and the results of an initial temporary deployment of two seismometers at the Montezuma Hills site. Following the temporary array deployment, the project was suspended and the array removed in August of 2010.

\section{Natural seismicity characterization}

We reviewed currently available public information including 25 years of recorded seismic events, location of mapped faults and estimates of the stress state of the region. We have also reviewed proprietary geological information collected by Shell, including seismic reflection imaging in the area, this information was reported in Myer, et al, 2010. There are known faults in this area, the one closest to the proposed injection site is the Kirby Hills Fault. The Kirby Hills fault is associated with earthquakes which are deep (9-17 miles below the surface) with magnitudes up to 3.7 (in 30+ year study period). The Shell data also indicates two unnamed faults in the area. The seismic events (earthquakes) we reviewed were not well located because of lack of nearby seismic stations, especially to the north and east. Therefore, attributing the recorded earthquakes to any single fault is inexact. This was somewhat unexpected given the relatively dense monitoring in California, but the Montezuma Hills site is on the very eastern edge of local networks, which are focused on the San Francisco Bay Area and the San Andreas Fault System. Figure 1 shows the seismic monitoring stations of the Northern California and 
Berkeley monitoring networks. Because of the relatively poor coverage, we revisited the historical events including visually inspecting seismograms and re-picking arrival times of seismic waves.

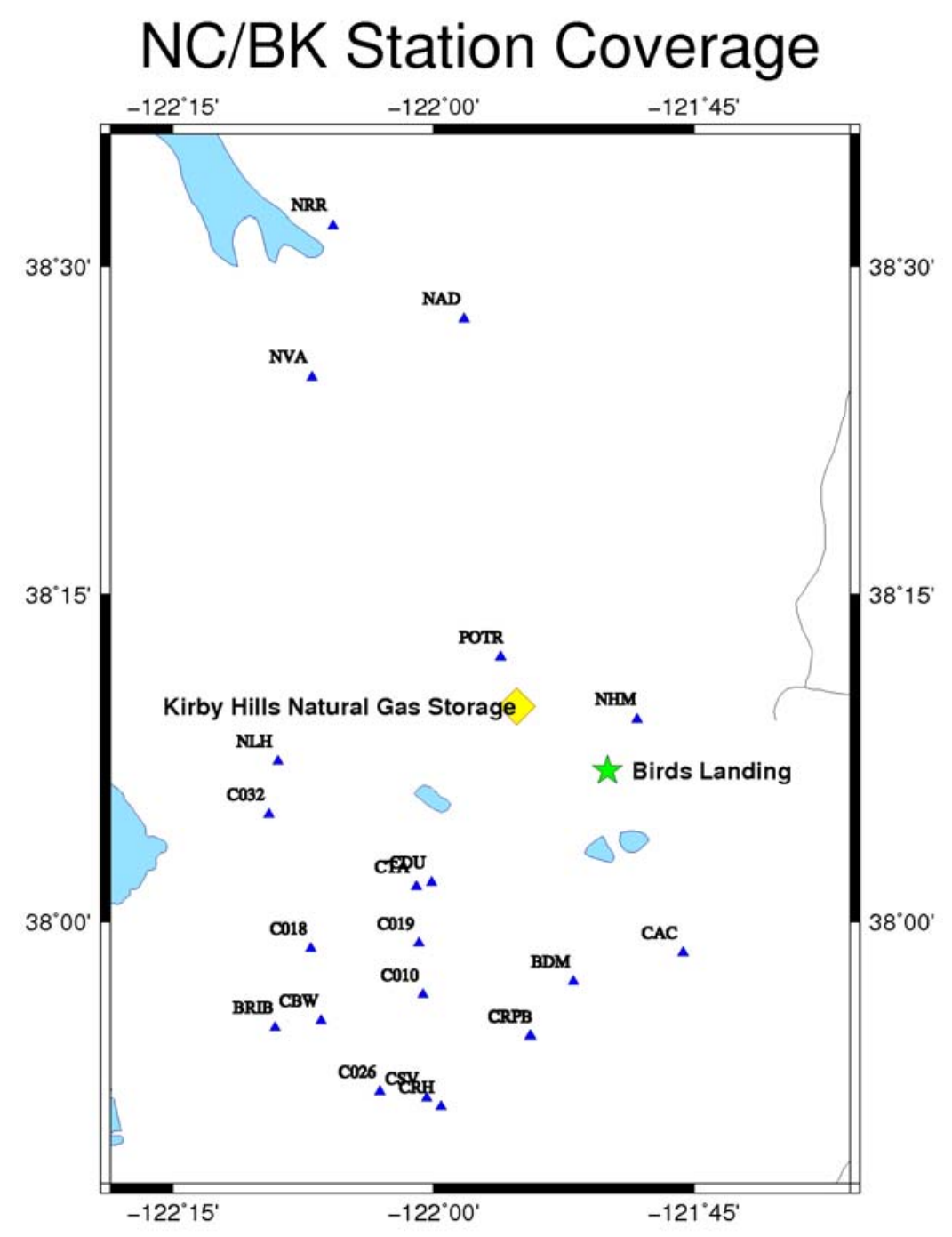

Figure 1. Station locations near the Montezuma Hills (Birds Landing) site for the Northern California Seismic Network (NCSN) and the Berkeley Digital Seismic Network. These stations were used for the event relocation.

We attempted to re-relocate all 111 earthquakes that were listed in the NCSN catalog to have occurred within a $11 \mathrm{~km}$ x $14 \mathrm{~km}$ rectangular area around Birds Landing from 1978 to the present. We also modified the general NCEDC northern California velocity model to a published velocity model specific to the area (Rhie and Dreger). We used HYPOINVERSE to re-locate the earthquake (Klein, 1985). The area and original locations obtained by the NCEDC (red dots) are shown in Figure 2. 


\section{Birds Landing Seismicity-Hypolnverse}

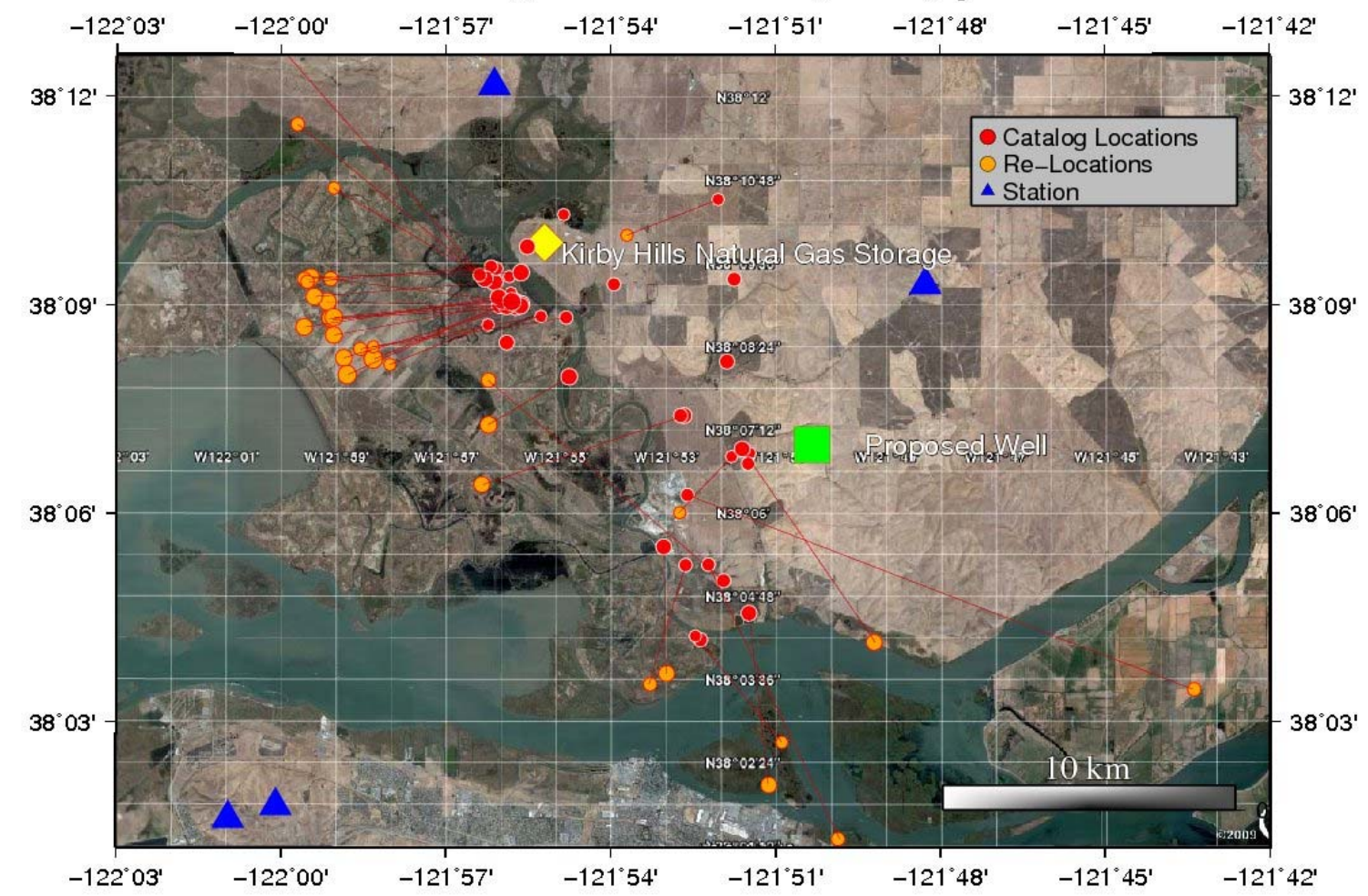

Figure 2. The original NCSN and Berkeley locations for events in the Montezuma Hills area (red) with lines connecting them to the new locations (orange). The green square is the proposed injection well location. Also shown are seismic stations (blue triangle) and the Kirby Hills underground injection facility for natural gas storage.

We obtained waveforms for 80 events for re-location. Of the 80 events, only 56 had sufficient waveform data for us to re-locate. We hand picked these data. We found that many phases were not identified by the auto-picker, but those that were auto-picked appeared to be fairly accurate. When we re-located the events using hand picks, the events moved considerably (up to $10 \mathrm{~km}$ ), and most moved outside the box. Re-located events are shown as orange dots in Figure 2. One consideration is that if all the events in the region were re-located, many that originally fell outside the box would move into the box.

\section{Temporary monitoring array design and deployment}

Because of the number and size of events in the area, we decided to deploy a monitoring network in advance of any subsurface injection. The initial step in the network deployment was installation of two temporary stations to assess data quality. The initial array design was considering both spatial sampling and a focus on the Kirby Hills fault west of the injection site. Figure 3 shows the location of the two temporary sites (MH-1 and $\mathrm{MH}-2$ ) along with potential locations for the 5 semi-permanent stations. The temporary site locations were put on property 
with ease of access and permitting, rather than by scientific design. Because the project was suspended, no further work on array design has been undertaken.

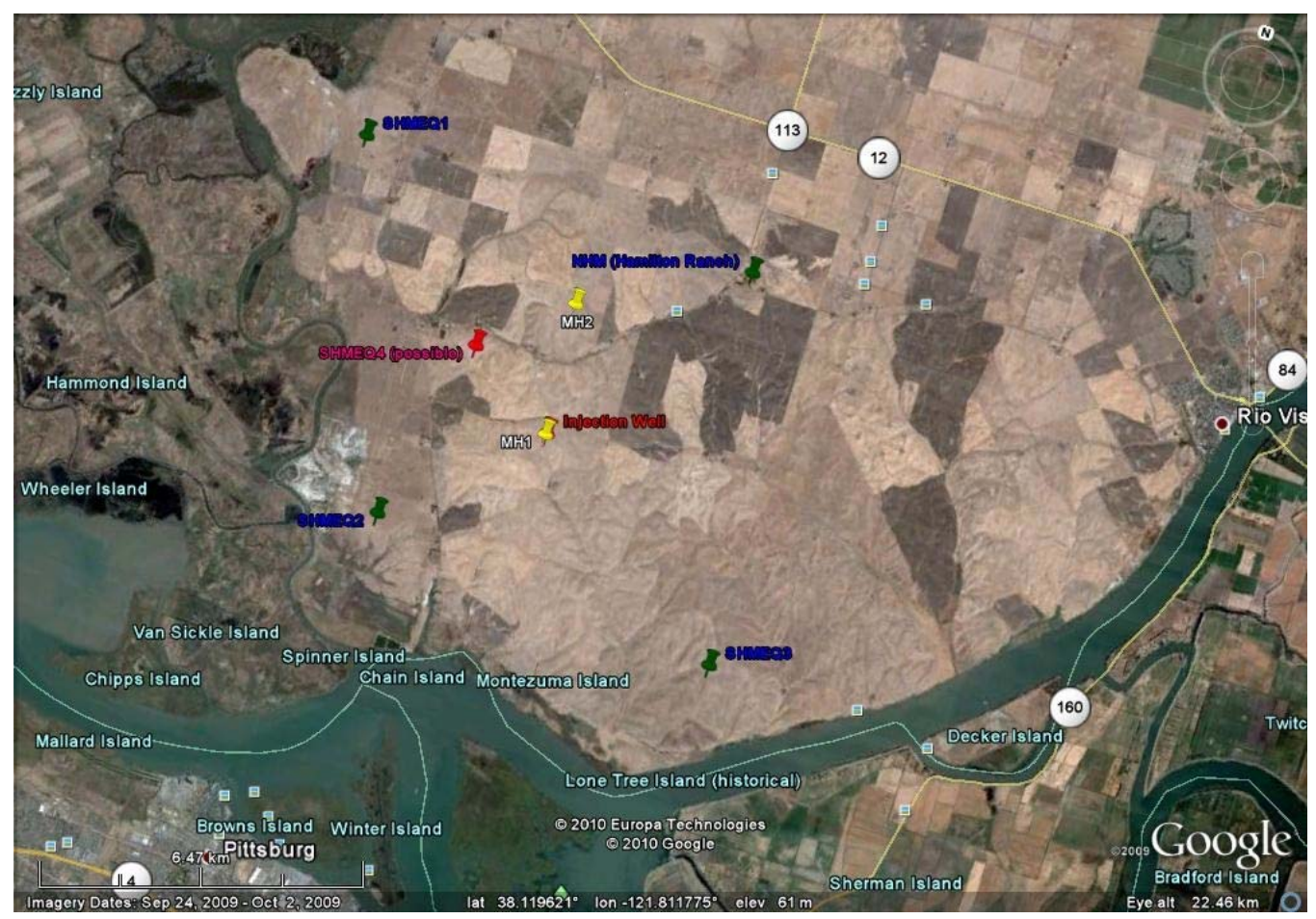

Figure 3. Locations of the temporary seismic stations (yellow markers) and potential stations (green and red markers), along with the injection well location. The town of Rio Vista is on the Eastern edge of the map and Pittsburgh, CA, is to the Southwest.

\section{Observations of the temporary microseismic array at Montezuma Hills}

Both temporary stations were deployed close to gravel access roads due to the agricultural use of the area. The station MH-1, accessed using Gate 1, operated from Day 138 to Day 230 (May 18 to August 18). In addition to the continuous acquisition, it also acquired triggered data starting on Day 173 (June 22). The station MH-2, accessed using Gate 3, was also deployed on Day 138 (May 18) but began have problems on Day 182 (July 2). Limited data are available after that. Figure 4 shows a photograph of a site. 


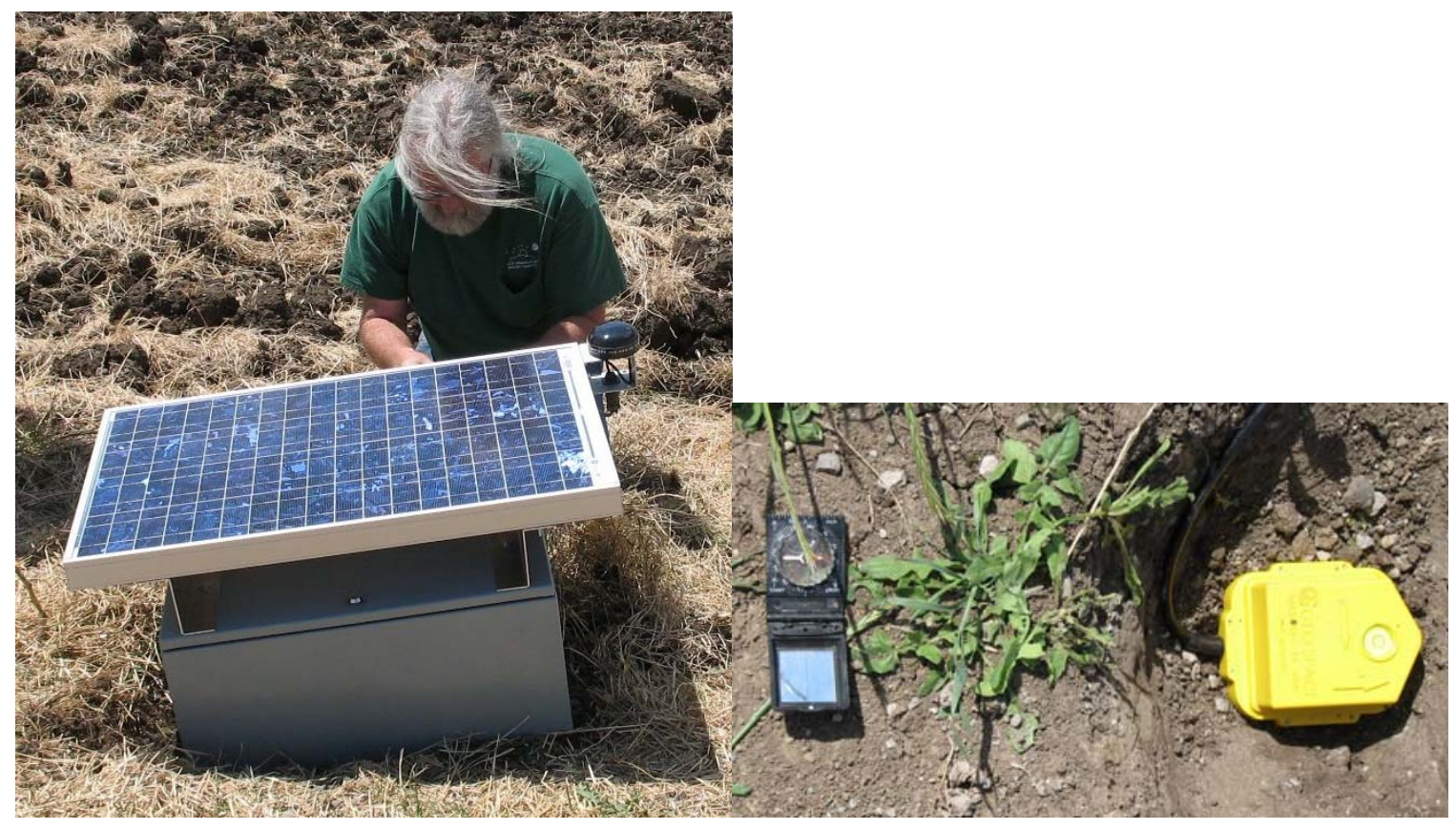

Figure 4. (left) A temporary seismic site with solar panel and recording system (inside grey box). (right) A three-component seismometer, placed about $3 \mathrm{~m}$ from the station in shallow hole, before being covered with dirt, along with compass used for alignment.

The data was acquired by a seismic recorder manufactured by Refraction Technology (REF TEK) which includes a global positioning system (GPS) clock for accurate time keeping. We recorded data from each station continuously. The REFTEK format data had a sample rate of 500 samples/second. Each file contained one hour of data (3600 seconds), and therefore 1,800, 000 samples. The data was converted to SEG-Y format (defined by the Society of Exploration Geophysicists). Each station had three components, so the SEG-Y file has three traces each $1,800,000$ samples long. Standard SEG-Y format limits the data to 32767 samples, so the data needed to be parsed to one minute trace lengths (30,000 samples). These seismograms are scanned manually for events. There were very few discrete events. Noise events were observed, many of which we interpret as being related to traffic on the gravel road. There were also smaller events that did not have characteristics of microseisms, but at 2-4 seconds length, seem to be too short for road traffic. An example of these is shown in Figures 5. These events are characterized by no impulsive onset and a relatively slow ramp up of energy, with an equally slow drop in energy. Figure 6 is another event which has an impulsive onset, but still does not look like a microseism. None of these 'noise' events were correlated between the two stations, meaning that even if they were true earth seismic events, they were very small and localized. The background noise at this site was expected to be influenced by commercial windmills which were operating near both stations. However no records of on/off times for the windmills were available, so we can not characterize the windmill noise specifically. Figure 7 show examples of background noise in the frequency domain (spectral content). 

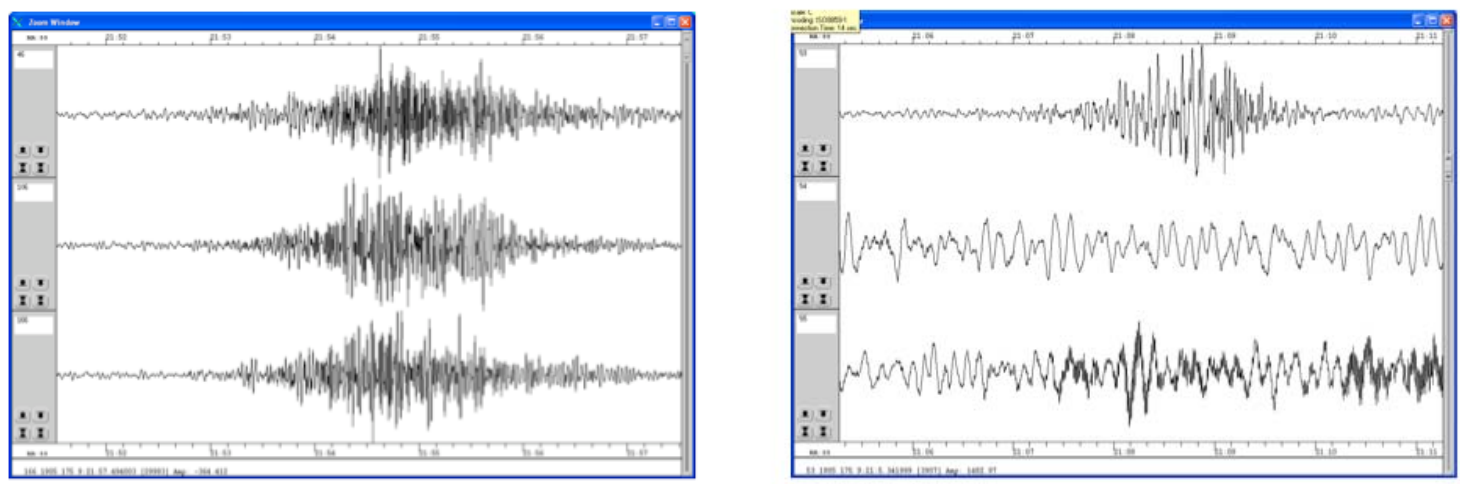

Figure 5. Two typical noise events which are not interpreted as earthquake events because of the lack of clear P- and S-wave arrivals and the non-impulsive onset.

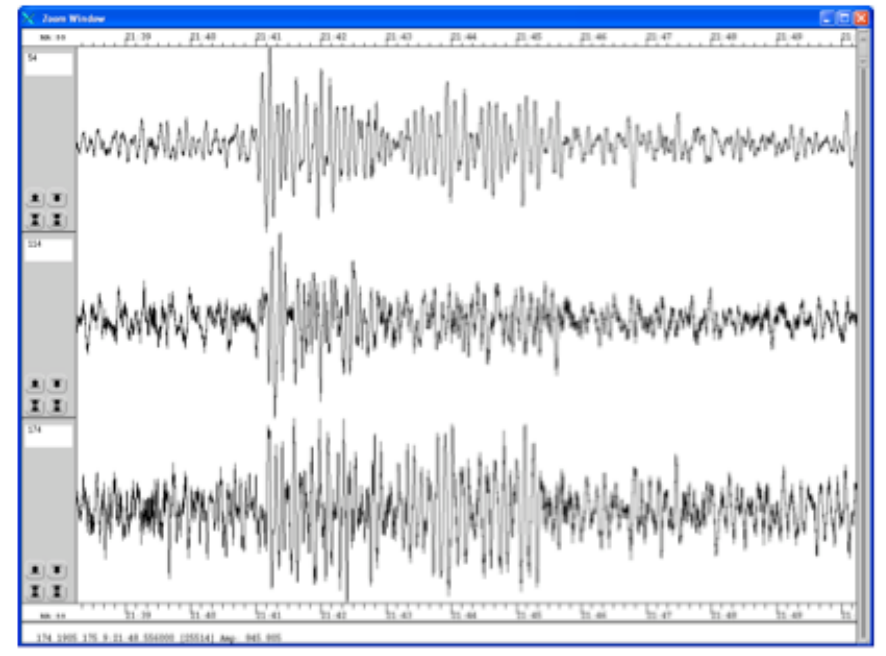

Figure 6. An impulsive event which is not interpreted as earthquake event because of the lack of clear S- wave arrival and the sudden end of high amplitudes. 


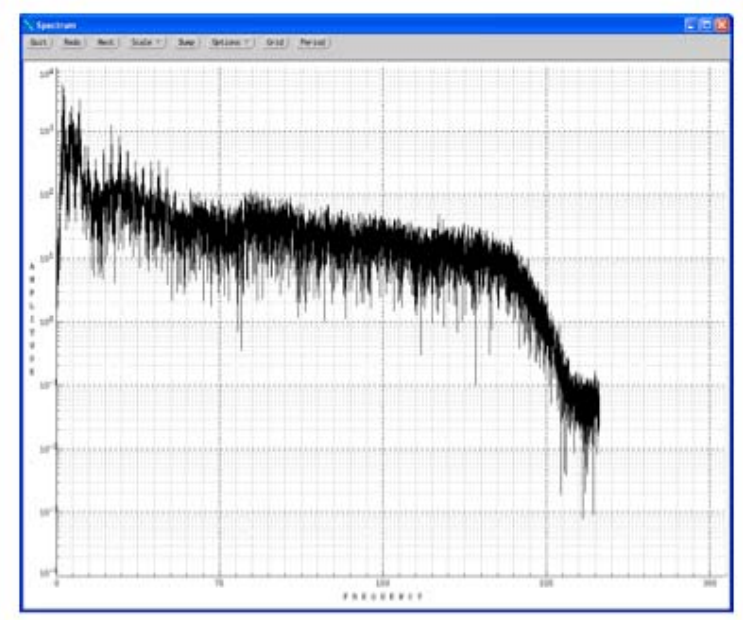

Figure 7. Spectral analysis of a noise recording showing the amplitude as a function of frequency.

One question addressed by the temporary stations was the ability of the REF TEK units to operate on 'triggered' event mode vs the continuous recording described previously. The triggered data worked very well. All of the triggered data were scanned and we found that every observable noise event was in the triggered data. This gives confidence that nothing would be missed if only the triggered data was recorded, which makes the identifying of events much more efficient. Also, any noise event was entirely recorded, no matter how long it was. The data for each day took about 5 minutes to manually scan, with some possibility of missing an event. There were between 0 and 10 triggers a day, with an average of 3 triggers, which means a month worth of triggers can be scanned in a few minutes. However, only one component of the data was recorded in triggered mode, so the continuous data is used in this report.

We searched the NCEDC catalog for events within 10 miles during the time of our temporary array deployment, and found 3 in the catalog (Table 1). There did not appear to be any observable events in our data at the time the first two catalog events. However, there was a clear event at the time of the third EQ (2010/06/21 22:29:06.80) shown in Figure 8.

Table 1. Events from the NCEDC data base during our deployment

\begin{tabular}{lllllll} 
Date & Time & Lat Lon & \multicolumn{2}{c}{ Depth Mag Magt Nst } \\
\hdashline 2010/06/08 & $16: 55: 52.90$ & $38.0920-121.9330$ & 22.93 & 1.78 Md 13 \\
2010/06/09 & $11: 05: 13.10$ & 38.1117 & -121.8777 & 18.97 & 1.59 & Md 10 \\
2010/06/21 & $22: 29: 06.80$ & 38.0785 & -121.8705 & 20.63 & 2.13 & Md 45
\end{tabular}



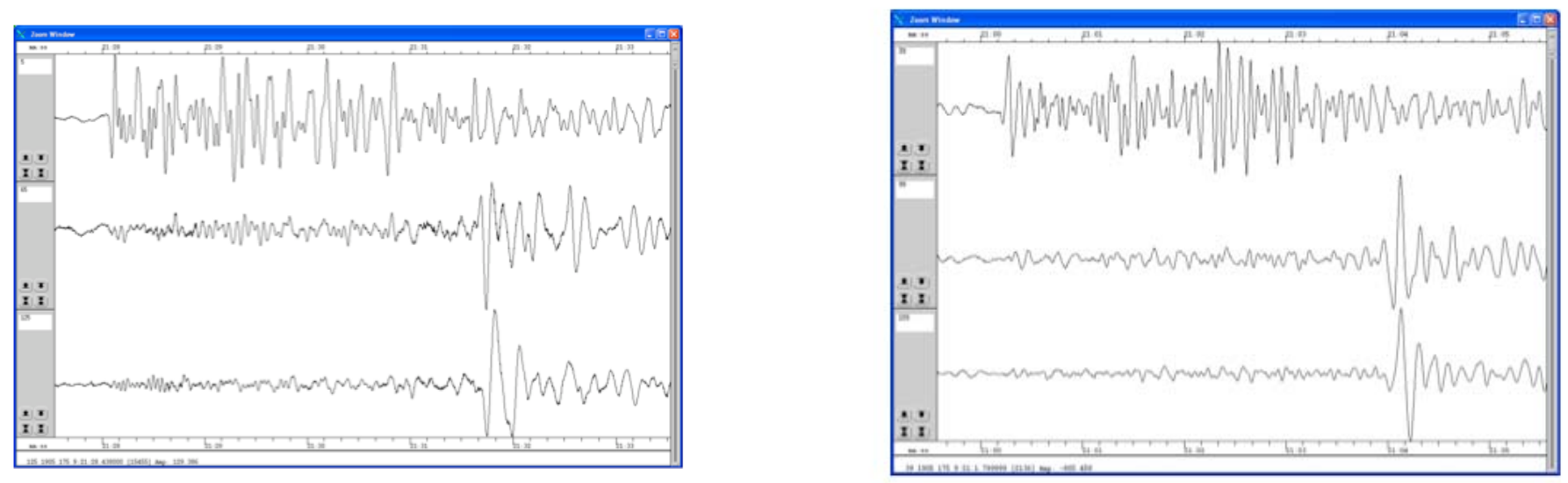

Figure 8. An earthquake event, identified as magnitude 2.1 from the NCEDC database, shown for station MH-1 (left) and MH-2 (right). The three seismograms are vertical, North and East (top, middle, and bottom, respectively).

\section{Conclusions}

Initial investigation of natural seismicity in the Montezuma Hills area found that the publicly available data sets were useful in characterizing historical seismicity, but that the locations of events in those databases were not very good for the study area. Our relocation of events showed a significant shift in locations. This highlights the need for dedicated monitoring stations designed for accurate locations in the area of study. The temporary array at Montezuma Hills was successful in characterizing noise sources, sensitivity and data recording parameters. At this point the study is suspended, however future work in the area will benefit from initial investigations.

\section{Acknowledgement}

This work was supported by the Office of Fossil Energy through the National Energy Technology Laboratory under Cooperative Agreement DE-FC26-05NT42593 with the California Energy Commission, and Lawrence Berkeley National Laboratory under U.S. Department of Energy Contract No. DE-AC02-05CH11231.

\section{References:}

Klein, F.W., 1985, HYPOINVERSE, a program for VAX and Pro-350 computers to solve for earthquake locations and magnitudes, U.S. Geological Survey Open File Report 85-515.

Myer, L., Laura Chiaramonte, Thomas M. Daley, Daniel Wilson, William Foxall, and John Henry Beyer, 2010, Potential for Induced Seismicity Related to the Northern California $\mathrm{CO}_{2}$ Reduction Project Pilot Test, Solano County, California, Lawrence 
Berkeley National Laboratory Report LBNL-3720E, Berkeley, California.

Larry R. Myer and Thomas M. Daley, 2010, Elements of a best practices approach to induced seismicity in geologic storage, Energy Procedia, in press.

Rhie, J. and Dreger, D.S., Waveform constrained seismic velocity structure in Northern California, (http://seismo.berkeley.edu/annual report/ar01 02/node34.html) 
Appendix 1.

Relocated Events

Date Time Lat Lon Depth Mag Magt Nst Gap Clo RMS SRC Event ID 1980/01/24 05:18:47.49 38.0708 -121.8657 18.12 2.63 Md 41108 11 0.16 NCSN 1983/06/09 04:05:51.24 38.1365 -121.8647 17.69 3.10 Md $10947 \quad 60.27$ NCSN 1983/09/20 00:35:11.24 38.1585-121.9360 $19.40 \quad 2.59$ Md $2591 \quad 12$ 0.19 NCSN 1984/10/28 11:35:46.61 38.0698 $-121.8727 \quad 18.27 \quad 2.84$ Md $46103 \quad 110.16$ NCSN 1986/04/05 17:32:42.90 38.1717 $-121.9142 \quad 17.55 \quad 2.52$ Md $33 \quad 64 \quad 100.19$ NCSN 1987/11/17 14:52:18.25 38.1562 -121.8625 $17.14 \quad 2.86$ 1988/06/20 01:15:58.30 38.1235 $-121.8777 \quad 21.05 \quad 3.20$ 1988/06/20 20:06:00.51 38.1235 -121.8787 21.012 .94 1989/09/11 16:20:35.74 38.0840 -121.8657 19.642 .87 1989/10/01 12:21:37.36 $38.1550-121.899016 .62 \quad 2.70$ 1989/10/01 13:10:24.28 38.1410 -121.9315 17.643 .00 1989/10/01 13:19:27.50 38.1640 -121.9252 15.593 .20 Md $6239 \quad 50.25$ NCSN Md $5060 \quad 70.15$ NCSN Md $4285 \quad 80.14$ NCSN Md $5560 \quad 100.14$ NCSN Md 7858270.25 NCSN ML 12135250.28 NCSN ML 14924280.37 NCSN 1989/10/01 21:41:58.64 38.1453 -121.9372 17.842 .54 1989/10/02 11:20:19.54 38.1470 -121.9135 21.562 .70 Md 3689120.14 NCSN Md $4061 \quad 100.13$ NCSN 1990/04/18 14:03:04.30 38.1137-121.8632 20.932 .52 1992/08/20 02:31:06.64 38.1328 -121.9125 20.183 .34 1992/11/23 20:59:55.56 38.0762 -121.8580 17.913 .26 1994/05/10 18:26:35.80 38.1045 -121.8767 20.942 .68 1994/07/11 18:25:48.81 38.0878 -121.8703 18.742 .71 1996/07/15 19:39:47.35 38.1145 -121.8577 21.642 .62 1996/07/15 21:44:36.35 38.1155 -121.8600 $21.14 \quad 3.22$ 1996/07/17 11:06:30.65 38.1120-121.8583 21.562 .79 1997/03/26 14:06:24.53 38.1568 -121.9307 22.742 .58 1997/03/26 15:34:59.51 38.1517 -121.9300 21.672 .81 1997/03/27 10:10:45.14 38.1507 -121.9287 21.553 .35 1997/03/27 10:26:35.30 $38.1492-121.9287 \quad 21.882 .92$ 1997/03/27 11:11:24.51 38.1505 -121.9268 $22.02 \quad 2.91$ 1997/03/27 11:30:06.99 38.1500 -121.9335 21.523 .57 1997/03/27 13:38:08.84 38.1498 -121.9273 $21.33 \quad 3.33$ 1997/03/27 14:01:24.23 38.1498 -121.9315 21.643 .48 1997/03/27 15:39:49.00 38.1510-121.9307 21.613 .70 1997/03/27 17:07:37.80 38.1528 1 -121.9302 $21.68 \quad 2.51$ 1997/03/27 17:16:42.79 38.1578 1 -121.9272 21.793 .41 1997/03/27 18:01:43.17 38.1555 -121.9352 $21.83 \quad 3.38$ 1997/03/27 22:16:18.77 38.1587 -121.9347 $21.68 \quad 2.65$ 1997/03/27 22:47:53.01 38.1510 -121.9328 21.863 .60 1997/03/27 22:53:07.62 38.1518 -121.9340 21.653 .46 1997/04/01 01:36:54.86 38.1508 -121.9300 21.573 .65 1997/04/01 11:25:54.45 38.1592 -121.9363 21.952 .73 1997/04/01 18:37:18.59 38.1563 -121.9382 21.563 .38 1997/04/02 12:14:12.37 38.1473 -121.9212 21.902 .51 1997/04/02 22:27:08.94 38.1572 $-121.9397 \quad 21.55 \quad 2.70$ 1999/04/04 18:12:15.38 38.0920 -121.8838 20.303 .19 2002/08/16 06:06:43.99 38.0877 -121.8772 20.412 .63 2007/03/05 21:26:56.29 38.0707 -121.8743 15.992 .52 2009/06/04 12:49:48.96 38.1753 -121.8673 21.212 .57 Md 1912270.15 NCSN Md 5281100.08 NCSN Md $5461 \quad 100.10$ NCSN Md 439790.10 NCSN Md $37108 \quad 90.06$ NCSN Md $37101 \quad 70.11$ NCSN Md $6790 \quad 70.12$ NCSN Md $5361 \quad 70.12$ NCSN Md $4596 \quad 110.12$ NCSN Md $48 \quad 65 \quad 110.06$ NCSN Md $57 \quad 86 \quad 110.06$ NCSN Md $48 \quad 85110.07$ NCSN Md $5186 \quad 110.06$ NCSN Md $6065 \quad 120.09$ NCSN Md $6165110.10 \mathrm{NCSN}$ Md $6086 \quad 110.08$ NCSN Mw $6357 \quad 110.08$ NCSN Md $5188 \quad 110.06$ NCSN Md $5490 \quad 110.08$ NCSN Md $60 \quad 89 \quad 120.08$ NCSN Md $5966 \quad 120.07$ NCSN Md 6165110.08 NCSN Md $6457 \quad 120.08$ NCSN Md $6372 \quad 110.07$ NCSN Md $5591 \quad 120.06$ NCSN Md $6454 \quad 120.10$ NCSN Md $3592 \quad 100.06$ NCSN Md $43 \quad 67 \quad 120.10$ NCSN Md $6258 \quad 100.13$ NCSN Md $4960 \quad 100.09$ NCSN Md 4359110.25 NCSN Md $7894 \quad 60.25$ NCSN 1050025 1096431 1103125 30821 69554 108533 119328 119319 143902 144828 144913 144978 144940 144873 156402 311727 326667 401972 30052630 30113343 30113368 30113545 499512 499523 499604 499607 499624 499625 499649 499650 499656 499679 499680 499681 499719 499729 499730 500112 500135 500154 500201 500230 21006629 21240822 40194201 40237628 


\section{DISCLAIMER}

This document was prepared as an account of work sponsored by the United States Government. While this document is believed to contain correct information, neither the United States Government nor any agency thereof, nor The Regents of the University of California, nor any of their employees, makes any warranty, express or implied, or assumes any legal responsibility for the accuracy, completeness, or usefulness of any information, apparatus, product, or process disclosed, or represents that its use would not infringe privately owned rights. Reference herein to any specific commercial product, process, or service by its trade name, trademark, manufacturer, or otherwise, does not necessarily constitute or imply its endorsement, recommendation, or favoring by the United States Government or any agency thereof, or The Regents of the University of California. The views and opinions of authors expressed herein do not necessarily state or reflect those of the United States Government or any agency thereof or The Regents of the University of California.

Ernest Orlando Lawrence Berkeley National Laboratory is an equal opportunity employer. 